\title{
Correction of static pressure on a research aircraft in accelerated flight using differential pressure measurements
}

\author{
A. R. Rodi and D. C. Leon \\ Department of Atmospheric Science, University of Wyoming, Laramie, Wyoming, USA
}

Correspondence to: A. R. Rodi (rodi@uwyo.edu)

Received: 9 April 2012 - Published in Atmos. Meas. Tech. Discuss.: 21 May 2012

Revised: 14 September 2012 - Accepted: 17 September 2012 - Published: 1 November 2012

\begin{abstract}
A method is described that estimates the error in the static pressure measurement on an aircraft from differential pressure measurements on the hemispherical surface of a Rosemount model $858 \mathrm{AJ}$ air velocity probe mounted on a boom ahead of the aircraft. The theoretical predictions for how the pressure should vary over the surface of the hemisphere, involving an unknown sensitivity parameter, leads to a set of equations that can be solved for the unknowns - angle of attack, angle of sideslip, dynamic pressure and the error in static pressure - if the sensitivity factor can be determined. The sensitivity factor was determined on the University of Wyoming King Air research aircraft by comparisons with the error measured with a carefully designed sonde towed on connecting tubing behind the aircraft - a trailing cone and the result was shown to have a precision of about $\pm 10 \mathrm{~Pa}$ over a wide range of conditions, including various altitudes, power settings, and gear and flap extensions. Under accelerated flight conditions, geometric altitude data from a combined Global Navigation Satellite System (GNSS) and inertial measurement unit (IMU) system are used to estimate acceleration effects on the error, and the algorithm is shown to predict corrections to a precision of better than $\pm 20 \mathrm{~Pa}$ under those conditions. Some limiting factors affecting the precision of static pressure measurement on a research aircraft are discussed.
\end{abstract}

\section{Introduction}

Static pressure measurement on an aircraft is inherently problematic because the pressure changes as the air accelerates around the wings and fuselage, as predicted by the Bernoulli equation. It is difficult to find a location on the aircraft to measure the true undisturbed pressure, $P_{\infty}$, i.e., the pressure at a distance far from the flow-disturbing effects. On the aircraft, static pressure $P_{\mathrm{S}}$ is measured at a set of ports where the aircraft designers have determined that the error, referred to as the static defect or position error, is minimal. In addition to causing errors in pressure-derived aircraft altitude, the static defect also leads directly to errors in airspeed and other measurements that need dynamic corrections, such as temperature, for example. In the pitot tube technique of airspeed measurement (Doebelin, 1990), dynamic pressure $q_{c}=P_{\mathrm{T}}-P_{\mathrm{S}} \simeq 1 / 2 \rho U^{2}$ is sensed, where $P_{\mathrm{T}}$ is the total pressure, $\rho$ the air density, and $U$ the airspeed. On the University of Wyoming King Air (UWKA) research aircraft, static pressure errors can be as large as $2 \%$ of $q_{c}$ at research aircraft speeds $\left(\sim 100 \mathrm{~m} \mathrm{~s}^{-1}\right)$, and this error transfers directly to an error of $1 \%\left(1 \mathrm{~m} \mathrm{~s}^{-1}\right)$ in airspeed. These errors directly affect estimates of atmospheric air motions which are sensed using the "drift method" in which the three-dimensional airspeed and ground velocity vectors are subtracted.

The static pressure ports on the UWKA are located on both sides of the fuselage near the rear of the aircraft. The ports are connected together in a manifold to compensate for the ram pressure effect that sideslipping can create on the upstream side of the fuselage. By manifolding, it is assumed that this effect will average to zero. But when lateral airspeed components occur because of turbulence, or by rudder application causing sideslipping, this assumption may not be correct, as is shown later in this paper. Further, these errors are likely to change with the deployment of wing flaps, landing gear, or the addition of external housings and fairings used to accommodate instruments. The usual approach is to develop corrections using data taken from flights past an instrumented tower, or from precise static pressure sources towed behind the aircraft (Brown, 1988; Wendisch and Brenguier, 2013). 
Static pressure becomes more problematic when aircraft are used in the study of pressure fields in baroclinic zones or cloud systems. Bellamy (1945) introduced airborne determination of $\mathrm{D}$-value, the difference between the radardetermined geometric altitude and the pressure altitude from static pressure, using the standard atmosphere assumption. Shapiro and Kennedy (1981) and Brown et al. (1981) applied this to the determination of jet stream geostrophic and ageostrophic winds. This pressure gradient approach has also been used for studies of low-level jets (Rodi and Parish, 1988; Parish et al., 1988; Parish, 2000). LeMone and Tarleton (1986) and LeMone et al. (1988) used altitude derived from accelerometer measurements instead of radar altitude in perturbation pressure studies around clouds suggesting that accuracies of $20 \mathrm{~Pa}$ can be obtained, but only with substantial empirical corrections and carefully flown legs. More recently, Parish et al. (2007) and Parish and Leon (2012) demonstrated that Global Navigation Satellite System (GNSS, hereafter referred to as global positioning system GPS) data can resolve both pressure gradients and perturbations associated with mesoscale and cloud-scale systems.

In this study, we develop and test a method for estimating static defect using differential pressure measurements on the hemispherical leading surface of a Rosemount model 858AJ (hereafter R858) air velocity probe. The theoretical predictions for how the pressure should vary (presented in the Appendix) lead to a set of equations that can be used to solve the static pressure error in addition to the attack angle, sideslip angle and dynamic pressure. We first use trailing sonde data to determine the probe sensitivity factor, and then compare resulting error estimates with accurate altitude measurements from a GPS-aided inertial measurement unit (IMU), allowing for an independent check of the precision of the algorithm and an examination of the effects of aircraft acceleration.

\section{Retrieval of static pressure error from differential pressure measurements}

Bögel and Baumann (1991) describe a method of analysis of R858 measurements during pilot-induced maneuvers to estimate static pressure errors. Crawford and Dobosy (1992) describe the Best Aircraft Turbulence (BAT) differential pressure flow-angle probe which addresses the static pressure problem by averaging pressure on several ports. Here, we develop a method to predict the static pressure error directly by using pressure measurements from the R858 air velocity probe which, on the UWKA, is mounted at the tip of a boom, as shown in Fig. 1. The probe is a hemispherecylinder configuration in which a hemispherical surface is at the end of a $2.5 \mathrm{~cm}$ diameter, $12.5 \mathrm{~cm}$ long cylindrical section. Pressure measurements on ports in a hemispherical surface are used for airspeed and flow angle determination (Brown et al., 1983). There are 5 ports: one central port which approximates total pressure, two ports separated by

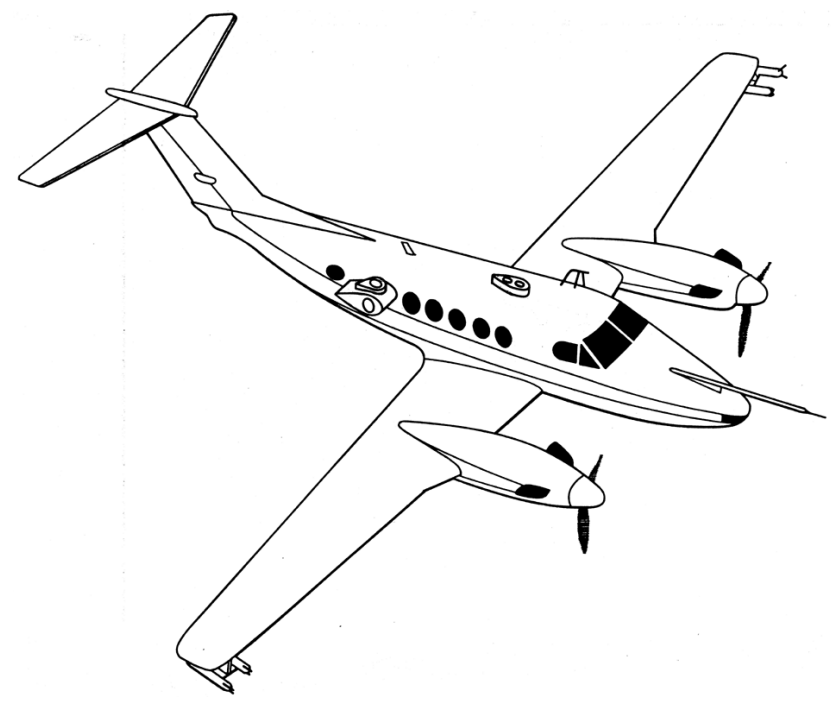

Fig. 1. UWKA showing nose boom location.

$\pm 45^{\circ}$ from the central axis in the aircraft horizontal plane for sideslip angle determination, and two ports separated by $\pm 45^{\circ}$ in the aircraft vertical plane for attack angle determination.

In the UWKA system, four differential pressure measurements are made, allowing the static error to be estimated. The method and specific equations used in the UWKA R858 configuration are presented in the Appendix. The attack angle $\alpha$, for example, can be estimated as described in the manufacturer's technical note (Rosemount, 1976) using

$\alpha \simeq \frac{P_{\alpha 1}-P_{\alpha 2}}{K q_{c}}$

where the numerator is the differential pressure between the two attack angle ports. The sensitivity coefficient, assuming potential flow (using a sensitivity factor, as defined in the Appendix, $f=9 / 4)$ for small angles, is $K \cong 2 f(\pi / 180)=$ $0.0785 \mathrm{deg}^{-1}$. Brown et al. (1983) investigated $K$ using data from flight maneuvers for the R858 on the noseboom on the NCAR Sabreliner. They found that for $N_{\text {Mach }}<0.5, K=$ $0.068 \mathrm{deg}^{-1}(f=1.95), 13 \%$ lower than the value recommended in Rosemount (1976). This determination of $K$ was not exact nor without ambiguities, however. The Brown et al. (1983) method substituted precise IMU-measured pitch angle for attack angle, which is valid during periods of straight and level flight assuming zero vertical wind and aircraft vertical velocity. Using pitch in this manner, the upwash effect (Crawford et al., 1996) of the fuselage, wings, and possibly the R858 itself is incorporated into the value of $K$. The sense of upwash effect is to make the local attack angle larger than the pitch angle, causing $K$ to be overestimated compared to the local value of $K$ without the upwash effect. Brown et al. (1983) apparently also used $\Delta P_{1}$ for $q$ without static defect or flow angle correction, which could further bias the Kvalues reported. In the present study, the sensitivity factor is 


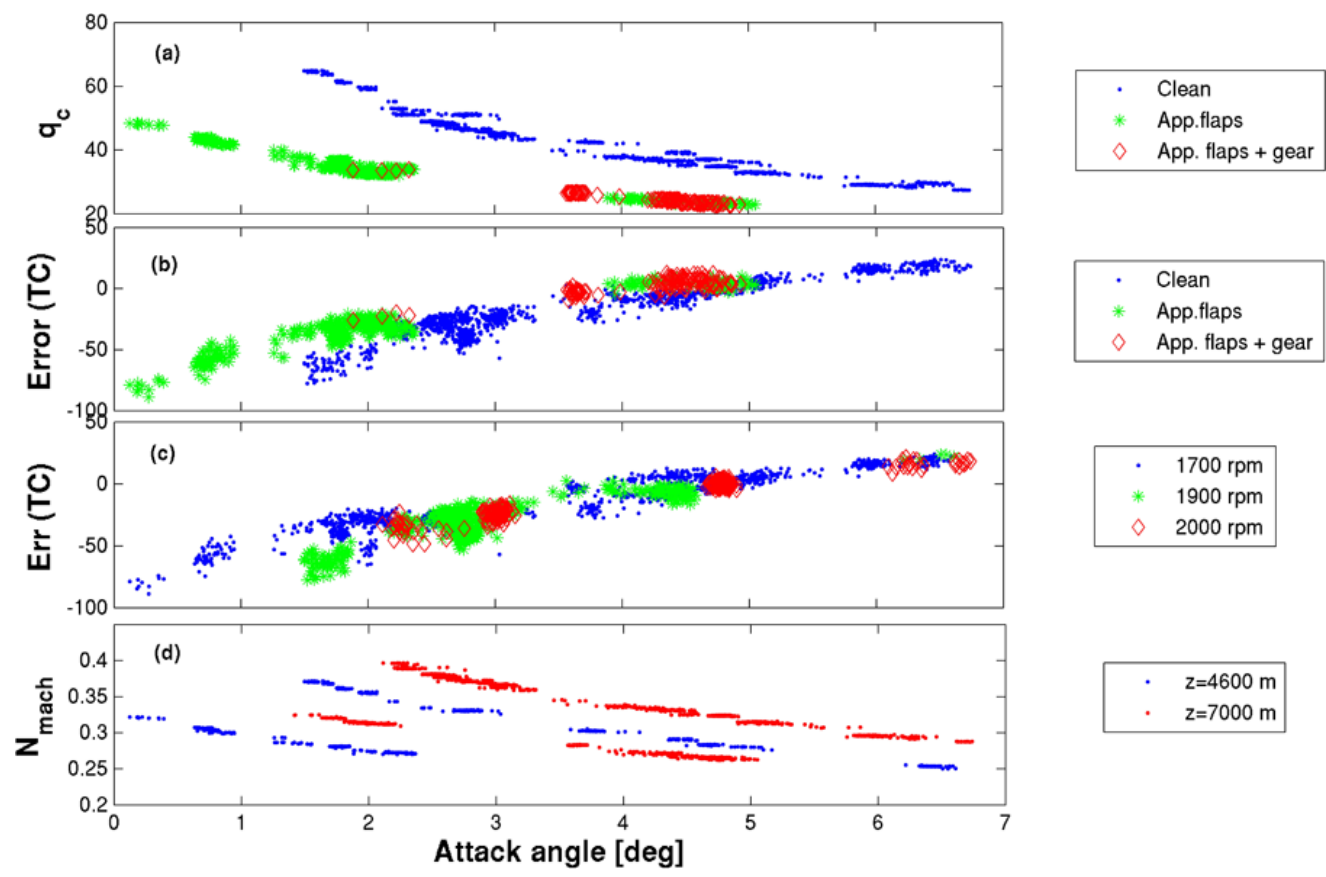

Fig. 2. Scatter plots $(10 \mathrm{~Hz}$ data) as functions of attack angle [deg] for various flight configurations and altitudes as indicated in legends: (a) dynamic pressure $q_{c}[\mathrm{hPa}]$, (b) and (c) static pressure error $[\mathrm{Pa}]$ determined by TC, and (d) Mach number.

estimated directly so that the upwash effect is not accounted for so that the flow angles that result are relative to the hemispherical surface, and $q$ is corrected for flow angle.

We now show that the static pressure error can be determined from the differential pressure measurements, assuming that sensitivity factor $f$ can be determined. The theoretical predictions of the four differential measurements from the Appendix (Eq. A11) are:

$$
\begin{aligned}
\Delta P_{1}=P_{1}-P_{\mathrm{s}, \mathrm{m}}= & q\left[1-(f-1)\left(\tan ^{2} \alpha+\tan ^{2} \beta\right) /\right. \\
& \left.\left(1+\tan ^{2} \alpha+\tan ^{2} \beta\right)\right]-P_{\mathrm{err}} \\
\Delta P_{\alpha}=P_{4}-P_{5}= & 2 f q \tan \alpha /\left(1+\tan ^{2} \alpha+\tan ^{2} \beta\right) \\
\Delta P_{\beta}=P_{2}-P_{3}= & 2 f q \tan \beta /\left(1+\tan ^{2} \alpha+\tan ^{2} \beta\right) \\
\Delta P_{R}=P_{1}-P_{2}= & f q\left(1-2 \tan \beta-\tan ^{2} \beta\right) / \\
& 2\left(1+\tan ^{2} \alpha+\tan ^{2} \beta\right)
\end{aligned}
$$

where here we have replaced $P_{\infty}$ in Eq. (A11) with the measured static pressure $P_{\mathrm{s}, \mathrm{m}}$ less the unknown error $P_{\mathrm{err}}$, and the remaining unknowns are the attack angle $\alpha$, sideslip angle $\beta$, dynamic pressure $q$, and the probe sensitivity $f$.

We note that we are solving for the error, using the expression for $\Delta P_{1}$ in Eq. (2) rearranged as

$$
\begin{aligned}
P_{\text {err }}= & q\left[1-(f-1)\left(\tan ^{2} \alpha+\tan ^{2} \beta\right) / .\right. \\
& \left.\left(1+\tan ^{2} \alpha+\tan ^{2} \beta\right)\right]-\Delta P_{1} .
\end{aligned}
$$

We show in the Appendix that $\alpha$ and $\beta$ can be found without a priori knowledge of $f$. Thus, $P_{\text {err }}$ is the departure of $\Delta P_{1}$ from $q$ after the attack and sideslip angle correction. The strategy is to develop an estimate $f$ using the trailing
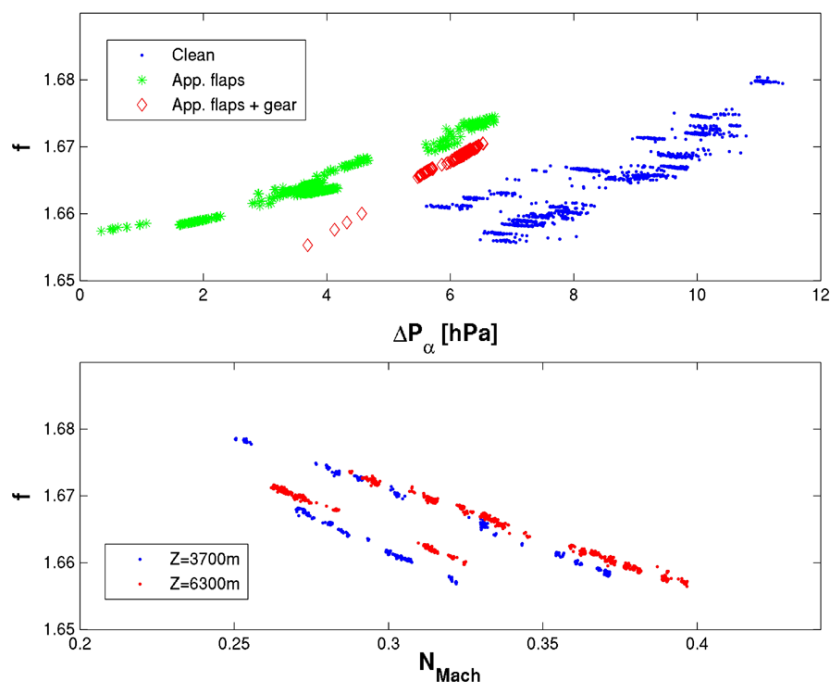

Fig. 3. Scatter plots $(10 \mathrm{~Hz}$ data) of probe sensitivity factor $f$, calculated from the solution of Eq. (2), versus $\Delta P_{\alpha}[\mathrm{hPa}]$ (top panel), and Mach number (bottom panel) for various flight configurations and altitudes, as indicated in legend.

cone test data, described in the next section, and then estimate $P_{\text {err }}$ as one of the unknowns from the four pressure measurements. 


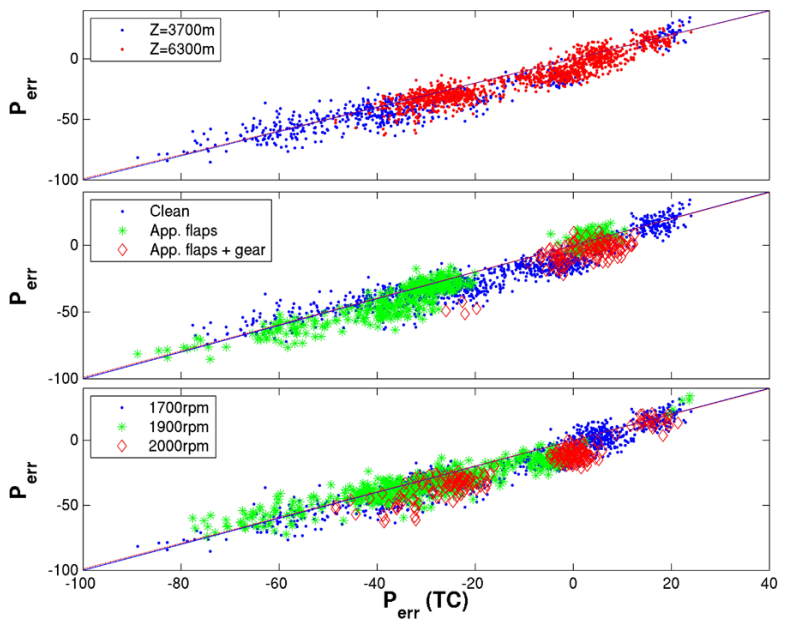

Fig. 4. Pressure error $[\mathrm{Pa}]$ predicted from differential pressure algorithm vs. error measured with TC for various flight configurations, altitudes and propellor speeds, as indicated in legends $(10 \mathrm{~Hz}$ data). $1: 1$ lines are shown.

\section{Trailing cone test data}

Ikhtiari and Marth (1964) and Mabry and Brumby (1968) describe an aircraft pressure calibration system in which a static pressure probe, connected to the aircraft by tubing, is towed at some distance behind and below the aircraft, away from the disturbing influence of the aircraft. The towed sonde is stabilized in flight by means of a carefully engineered cone - thus the term "trailing cone" (hereafter called TC ) - that creates drag for stabilization of static pressure ports which are distributed around the circumference of a straight piece of tubing behind the cone. Brown (1988) described the technique in detail, and demonstrated for the NCAR Sabreliner that the largest expected error in the pressure measurement is $\pm 39 \mathrm{~Pa}$ after application of corrections based upon the TC data. The distance that the TC is extended is chosen to be long enough to minimize pressure fluctuations in the aircraft's wake. However, accelerations cause errors. For example, with a $19 \mathrm{~m}$ tubing length (the length used for the UWKA), a $0.1 \mathrm{~g}$ acceleration will result in a $15 \mathrm{~Pa}$ effect. Consequently, we limit data collection to steady, straight flight legs.

A UWKA test flight using the Douglas model $501 \mathrm{TC}$ was conducted on 27 October 2005. Data were collected in several configurations: (1) with the aircraft in "clean" configuration (landing gear raised, flaps retracted), (2) in segments with gear lowered, (3) with gear lowered and flaps extended in approach mode, and (4) at different power settings and altitudes. Several measured variables are shown in Fig. 2 as a function of aircraft attack angle, using data filtered at $5 \mathrm{~Hz}$ and output at $10 \mathrm{~Hz}$. The lack of a single relationship for all configurations among dynamic pressure, pressure error, and $N_{\text {Mach }}$ is evident. This is the main obstacle to simple corrections as a function of one variable such as dynamic pressure or attack angle.

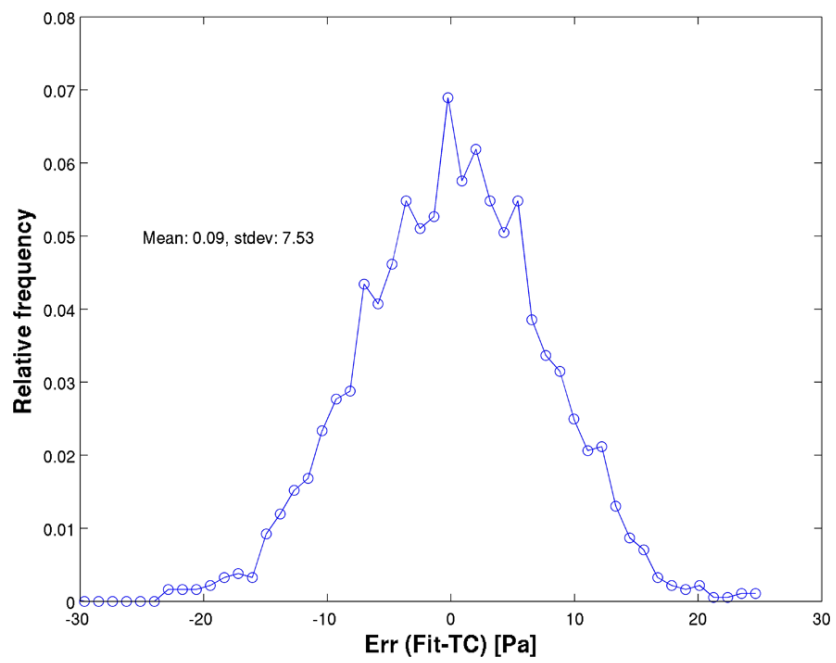

Fig. 5. Distribution of residual error (DP algorithm prediction minus TC measurement) $[\mathrm{Pa}](10 \mathrm{~Hz}$ data).

\section{Empirical determination of $f$ from trailing cone data}

We first solved Eq. (2) for the unknown values of $f, \alpha, \beta$, and $q_{c}$ using TC flight measurements of $P_{\text {err }}$ and differential pressures $\Delta P_{1}, \Delta P_{\alpha}, \Delta P_{\beta}$, and $\Delta P_{R}$. The resulting $\mathrm{f}$ values are plotted in Fig. 3, and show increases with $\Delta P_{\alpha}$, and decreases with $N_{\text {Mach. }}$. We then used a non-linear regression procedure to obtain the empirical estimate of $f$ that best predicts the TC-determined $P_{\text {err }}$. The best fit was found by trial and error selection of variables to be:

$f=c_{0}+c_{1} N_{\text {Mach }}+c_{2} N_{\text {Mach }}^{2}+c_{3} \Delta P_{\alpha}$

where the constants were found to be $c_{0}=1.700, c_{1}=$ $-0.1569, c_{2}=0.06633$ and $c_{3}=+0.001254$, and where $\Delta P_{\alpha}$ is in units of $\mathrm{hPa}$. A discussion of the physical basis for these relationships will be presented later.

The resulting predictions of pressure error are compared with TC measurements in Fig. 4 at various steady flight configurations and power settings. The distribution of the residual errors is shown in Fig. 5 to have a precision $\pm 10 \mathrm{~Pa}$ in all configurations ( $\sigma=8 \mathrm{~Pa})$.

There is additional evidence for the variability of $f$. Brown et al. (1983) and Rosemount (1976) provide experimental evidence for $f$ decreasing with $N_{\text {Mach }}$, consistent with the present results. Traub and Rediniotis (2003) (TR03) present an analytical prediction of surface pressures for a hemisphere-cylinder configuration similar to the R858, and wind tunnel results at Reynolds numbers about a factor of two higher than UWKA flight $\left(N_{R e} \approx 1.5 \times 10^{4}\right)$. The TR03 theoretical formulation, confirmed by their wind tunnel results, predicts sensitivity to be $f=2.07$ at zero incidence angle, and also their data show that the sensitivity varies with incidence angle.

To explore the effect of probe shape on $f$, we used a commercially-available finite-element solver for turbulent, 


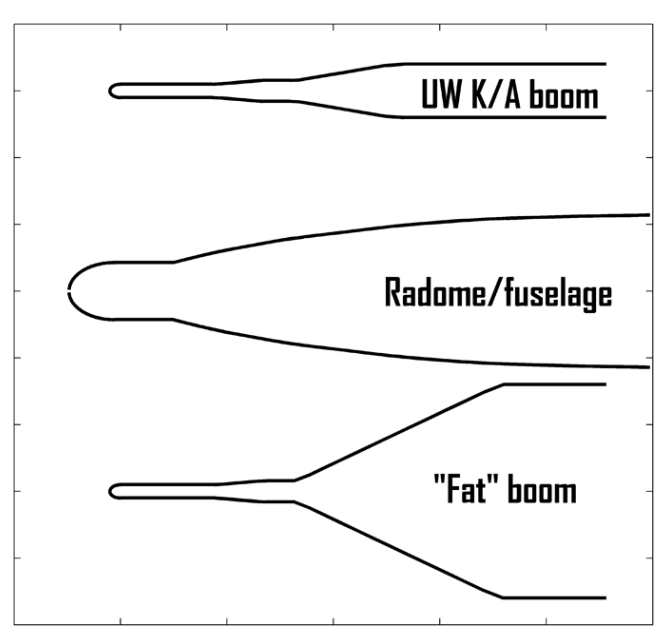

Fig. 6. Models of aircraft and boom configurations (see text).

compressible flow equations (FIDAP, Fluid Dynamics International, Inc.). An axisymmetric, compressible model at zero attack angle was used and showed that $f$ is different for various mounting configurations, and changes with speed. Solutions were found for $N_{\text {Mach }}$ equivalent to speeds of 50$180 \mathrm{~m} \mathrm{~s}^{-1}$. Four configurations were modeled as shown in Fig. 6 (not to scale): (1) a sphere (not shown); (2) the R858 probe mounted on the end of the UWKA nose boom, which is $0.2 \mathrm{~m}$ in diameter; (3) the spherical radome (nose radar covering) as on the former National Science Foundation (NSF) King Air aircraft (N308D); and (4) the R858 mounted on a nose boom four times the diameter of the actual UWKA nose boom. Pressure distributions on the spherical surface were fit using the $\sin ^{2}$ relationship (A1), and the resulting $f$ for each $N_{\text {Mach }}$ plotted in Fig. 7a. For the sphere, $f$ varies from 1.9-2.1. Adding the nose boom behind the R858 hemisphere-cylinder decreases $f$ to about 1.9. Increasing the boom diameter to $0.8 \mathrm{~m}$ lowers $f$ to about 1.5 . These results suggest that there is no unique value of $f$ for all mounting configurations of the R858. Indeed, the fuselage of the aircraft itself presents a formidable aerodynamic barrier to the probe, which is likely to contribute to the actual $f$ variability in addition to a particular mounting configuration.

To explore further the behavior of $f$, we constructed a physical model of the R858 with extra pressure ports drilled so that adequacy of the $\sin ^{2}$ relationship between angle and pressure could be determined by direct measurement along with determination of $f$. The test was conducted in the University of Wyoming Low Speed Wind Tunnel, which has a test section of $0.6 \times 0.6 \times 0.9 \mathrm{~m}$. The model was constructed $75 \%$ larger than the actual probe so that the Reynolds number at the test speed of $50 \mathrm{~m} \mathrm{~s}^{-1}$ would be approximately that of the actual $(0.0254 \mathrm{~m}$ diameter $)$ probe at $90 \mathrm{~m} \mathrm{~s}^{-1}$, the typical flight speed for the UWKA. Data were digitized with a personal-computer-based data logging system at $10 \mathrm{~Hz}$ after analog filtering with cutoff frequency of $2 \mathrm{~Hz}$.
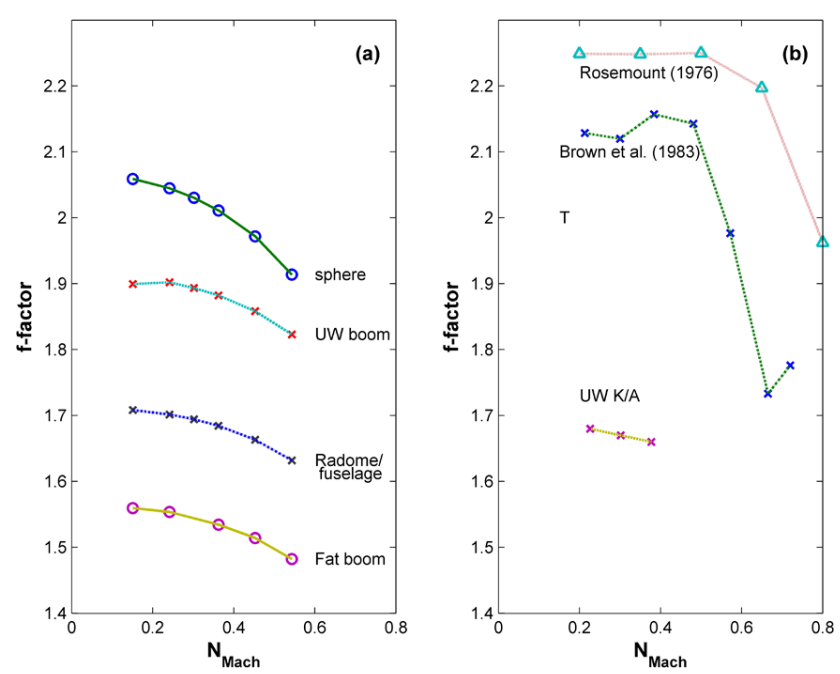

Fig. 7. Estimation of sensitivity factor $f$ from (a) modeling and (b) values from Brown et al. (1983), (Rosemount, 1976), and solution to Eq. (A11) for UW King Air flight data. The point labeled " $T$ " represents the value from determined from the wind tunnel tests of the R858 model.

The analysis then was performed on one minute averages of pressures at each port. Additional measurements made with a standard pitot tube placed upwind from the test body to ensure that the tunnel speed did not change during runs at each attack angle. In Fig. 7b, the sensitivity of $f$ to $N_{\text {mach }}$ from Brown et al. (1983) and (Rosemount, 1976) are shown, along with $f$ determined from the wind tunnel tests (point labeled as " $T$ "), and the solution of Eq. (2) with actual King Air flight data.

\section{Measured pressure compared to pressure derived from GPS altitude}

While the 2005 TC test flight varied attack angle with airspeed and altitude, sideslip angles were intentionally kept near zero to prevent lateral acceleration. In this section, the efficacy of the estimates of $P_{\text {err }}$ in an accelerating flight environment using the differential pressure solution described in the previous section will be examined.

Differential global positioning system (dGPS) techniques use data from one or more stationary reference or base GPS stations which have precisely determined location to refine position estimates for the receiver on the aircraft. dGPS processing techniques using dual-frequency (L1/L2) carrier phase data can eliminate errors caused by ionospheric and tropospheric delays entirely, resulting in position estimates with accuracy of centimeters under optimal conditions (Parish et al., 2007).

In the present study, we use post-processed inertial measurement unit (IMU) data in conjunction with dual-frequency dGPS data to resolve the aircraft position and motion 


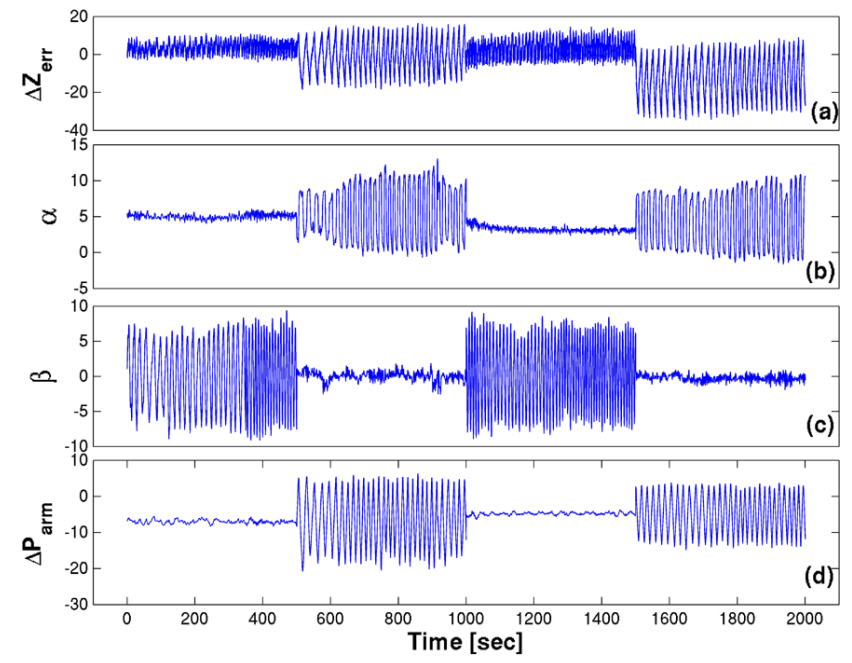

Fig. 8. Time series of (a) height difference (geometric-pressure) [m], (b) attack angle [deg], (c) sideslip angle [deg], and (d) pressure deviation from lever arm correction $[\mathrm{Pa}]$. Time series is the concatenation of four $500 \mathrm{~s}$ segments, the first two flown at $4600 \mathrm{~m}$, the second two at $7000 \mathrm{~m}$.

(Trimble/Applanix model AV410). The IMU data, recorded at $200 \mathrm{~Hz}$, were corrected in post-processing using Trimble/Applanix POSPac software which implements a tightlycoupled Kalman filter between the IMU and dGPS data. The processing fully removed all L1/L2 cycle ambiguities in a fixed, narrow lane processing mode. Position accuracy estimated by the manufacturer is shown in Table 1 . The resulting $200 \mathrm{~Hz}$ values of aircraft position and attitude were low-pass filtered with a cutoff frequency of $10 \mathrm{~Hz}$ and then decimated to $20 \mathrm{~Hz}$ for the present analysis. Also, accurate time synchronization of the IMU and pressure measurements was assured by GPS time stamping of all data.

Static pressure was measured with the Rosemount 1501 High Accuracy Digital Sensing Module (HADS) which has static accuracy of $20 \mathrm{~Pa}$ and a digital resolution of $1.8 \mathrm{~Pa}$. The accuracy includes effects of non-linearity, repeatability, temperature $\left(-51\right.$ to $\left.80^{\circ} \mathrm{C}\right)$ and calibration. Worst case error from transducer acceleration is specified to be $20 \mathrm{~Pa}$ under acceleration of $6 \mathrm{~g}$; the maximum acceleration in these tests was $\pm 1 \mathrm{~g}$. We estimate the maximum dynamic errors in the connecting tubing to be $10 \mathrm{~Pa}$ for longitudinal accelerations (here $0.1 \mathrm{~g}, 10 \mathrm{~m}$ tubing length) and lateral (1 g, $1 \mathrm{~m}$ tubing length) accelerations of the air column.

Flight data were collected on 16 September 2011 during pilot-induced maneuvers inducing variations in attack and sideslip angles. Periods of turns were not considered in the analysis. The aircraft was flown at nominally constant pressure with deviations corrected hydrostatically to that pressure using the method described by Parish et al. (2007). Pressurederived altitude changes were determined from integration of the hydrostatic equation:
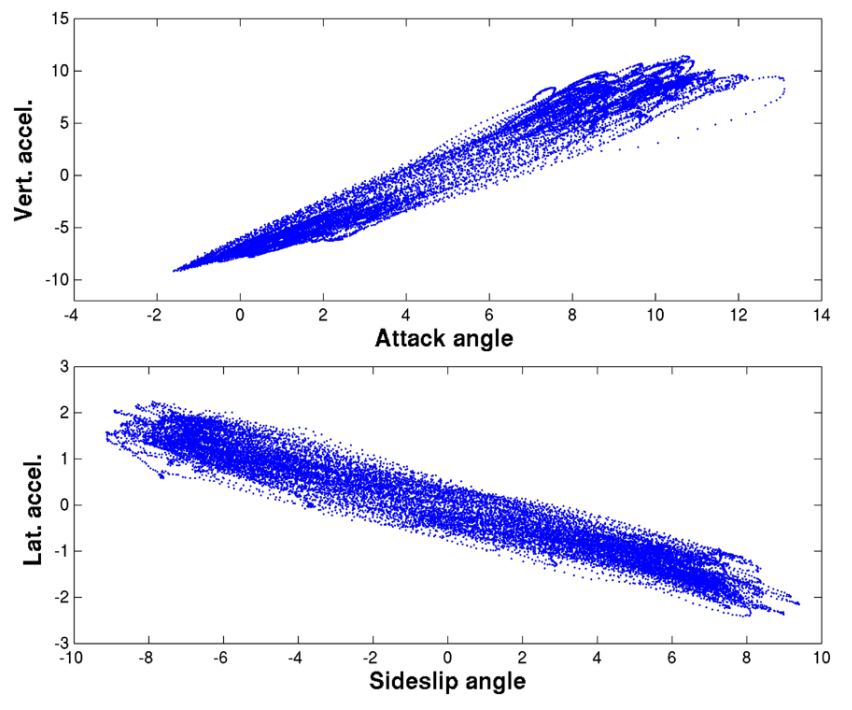

Fig. 9. Scatter plots of: (top panel) attack angle [deg] vs. vertical acceleration $\left[\mathrm{m} \mathrm{s}^{-2}\right]$; and (bottom panel) sideslip angle [deg] vs. lateral acceleration $\left[\mathrm{m} \mathrm{s}^{-2}\right]$.

Table 1. Trimble/Applanix airborne positioning system performance specifications.

\begin{tabular}{ll}
\hline AV410 Absolute Accuracy & Post-processed \\
\hline Position $(\mathrm{m})$ & $0.05-0.30$ \\
Velocity $\left(\mathrm{m} \mathrm{s}^{-1}\right)$ & 0.005 \\
Roll and Pitch $(\mathrm{deg})$ & 0.008 \\
True Heading $(\mathrm{deg})$ & 0.025 \\
\hline AV410 Relative Accuracy & \\
\hline Noise $\left(\operatorname{deg} \mathrm{h}^{-0.5}\right)$ & $<0.1$ \\
Drift $\left(\operatorname{deg} \mathrm{h}^{-1}\right)^{*}$ & 0.5 \\
\hline
\end{tabular}

* Attitude will drift at this rate up to the maximum absolute accuracy.

$z-z_{0}=-\int_{P_{0}}^{P} \frac{R_{\mathrm{dry}} T_{\mathrm{v}}}{g} \mathrm{~d} \ln P$

where $z_{0}$ is a direct measurement of geometric altitude from the IMU/dGPS system at pressure $P_{0}$ and virtual temperature $T_{\mathrm{V}}$. Data were collected at two altitudes - nominally 4600 and $7000 \mathrm{~m}$ m.s.l. Other relevant measurements include in-house developed reverse flow temperature (accuracy of $0.5 \mathrm{~K}$, resolution of $0.006 \mathrm{~K}$ ), and Edgetech Model 137 dew point temperature (accuracy of $1 \mathrm{~K}$, resolution $0.006 \mathrm{~K}$ ).

A bias is introduced if an atmospheric horizontal pressure gradient exists, or when pressure is falling, along the flight track since constant geometric height is no longer constant pressure. To minimize this effect, the time series is broken up into $500 \mathrm{~s}$ segments and reinitialized with $z_{0}$ from the highly accurate IMU/dGPS geometric altitude value at that instant. 


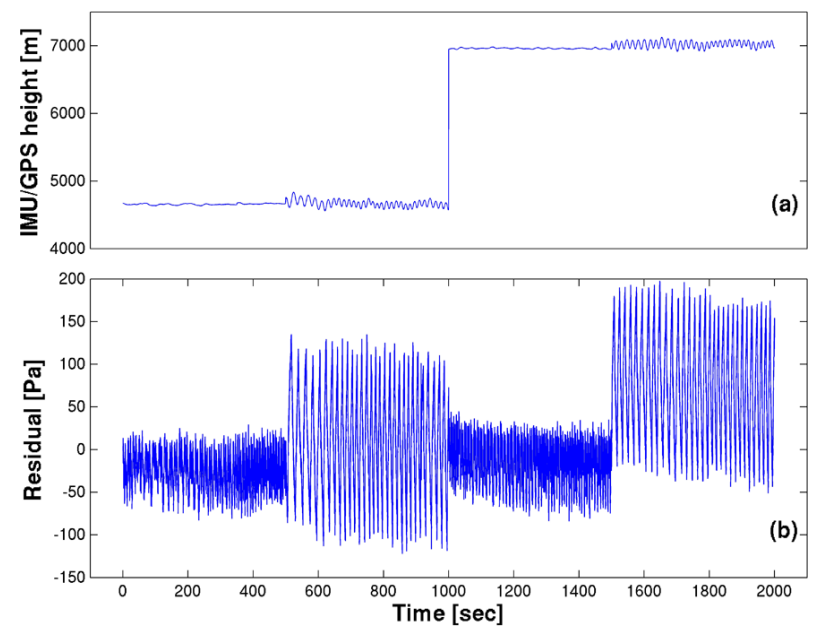

Fig. 10. Concatenated time series as in Fig. 8: (top panel) aircraft height $[\mathrm{m}]$ (m.s.l.); and (bottom panel) pressure error determined from IMU/dGPS before correction [Pa].

The Laramie Valley was under the influence of high pressure, clear sky, and weak pressure gradient during the analysis period, which minimized the pressure change effect.

Computing geometric altitude from measured static pressure also involved carefully considering the relative distance vectors of the inertial measurement unit (IMU), GPS antenna, R858, and the static pressure locations. These vectors were accurately determined with accuracy $<5 \mathrm{~cm}$ using precise surveying techniques. The resulting "lever arm" fluctuations were less than $8 \mathrm{~Pa}$ during the sideslip and $15 \mathrm{~Pa}$ during the attack angle changes, as shown in Fig. 8d.

The angle changes with periods of about $10 \mathrm{~s}$, as shown in Fig. 8b, result in correlated vertical accelerations as shown in Fig. 9. The attack angle was limited to -2 to $-12^{\circ}$, resulting in $\pm 1 \mathrm{~g}$ changes, helping to avoid excessively large altitude excursions. The sideslip angles (Fig. 8c) were restricted to $\pm 8^{\circ}$, limiting lateral accelerations to $\pm 0.20 \mathrm{~g}$ lateral accelerations for crew comfort and also safety considerations. Typical values during research flights are less than $\pm 0.5 \mathrm{~g}, \pm 1 \mathrm{~g}$ in strong turbulence, and only rarely experiencing the $\pm 2 \mathrm{~g} \mathrm{lim}$ itation set under U.S. Federal Aviation Administration Part 91 certification. In our experience, instantaneous attack angle values in severe turbulence have been noted to reach $20^{\circ}$ while the aircraft is still within the g-loading limits, but this is rare. The resulting static pressure errors from the maneuvers are shown in Fig. 10. The pressure-geometric altitude errors for sideslipping have a range of about $50 \mathrm{~Pa}$ and for attack angle changes a range of about $300 \mathrm{~Pa}$

The errors before and after correction are shown in Fig. 11 for the attack and sideslip changes, with the distribution of errors before and after correction shown in Fig. 12. After correction, the biases in during the attack and sideslip changes were -14 and $+10 \mathrm{~Pa}$, respectively, with standard deviations of 16 and $11 \mathrm{~Pa}$, respectively.
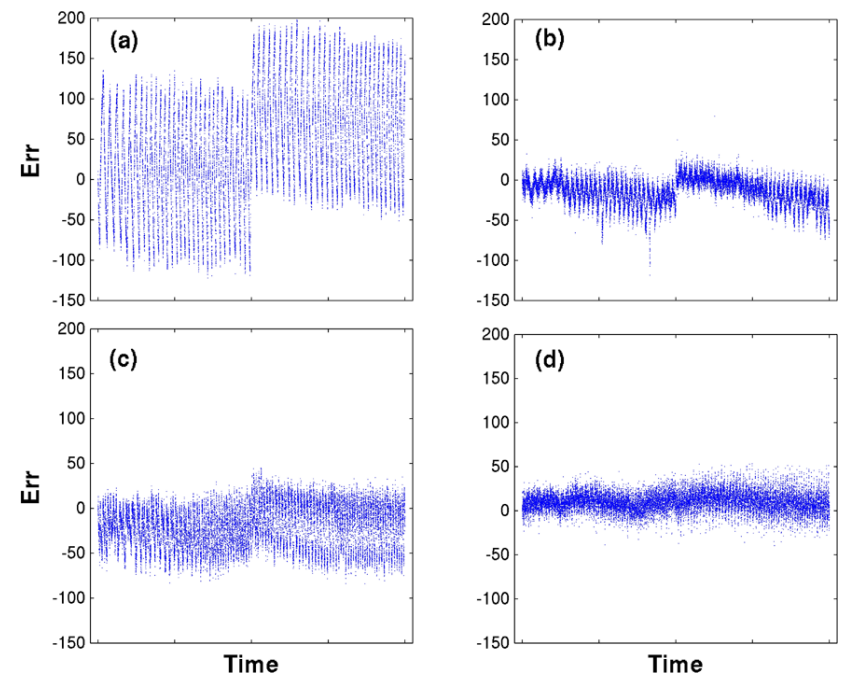

Fig. 11. Time series of errors [Pa] from Fig. 10: (a) and (b) are before and after correction, respectively, for attack changes; (c) and (d) before and after correction, respectively, for sideslip changes.
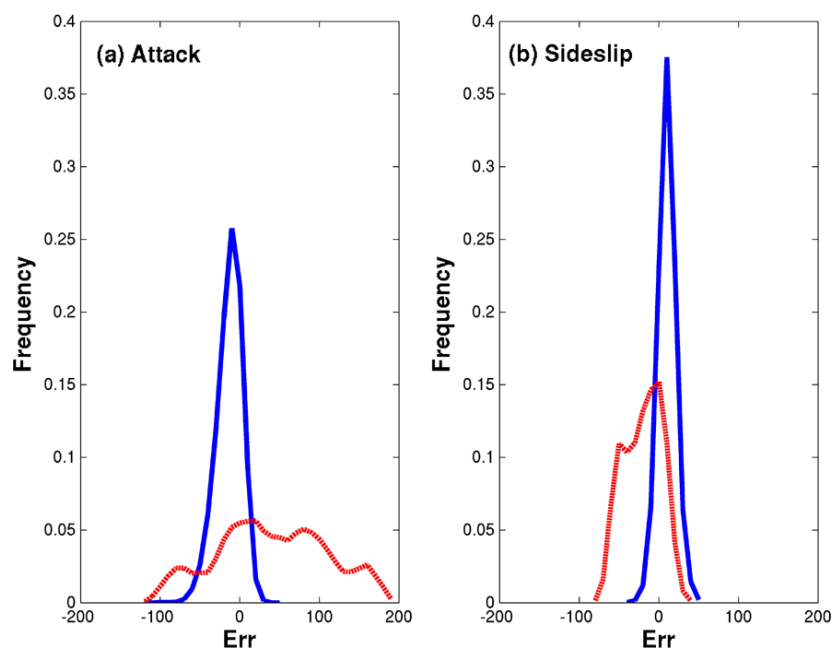

Fig. 12. Distribution of errors [Pa] for data shown in Fig. 11 before correction (red) and residual after correction (blue) for (a) attack angle changes; and (b) sideslip angle changes.

\section{Frequency response}

The power spectral density (PSD) plots of $100 \mathrm{~Hz}$ static pressure and correction data are shown in Figs. 13 and 14 for attack angle and sideslip angle changes, respectively. Data is from the $7000 \mathrm{~m}$ altitude in which the atmospheric turbulence is very low. Also shown on the plots is the vertical wind speed in these legs. The derived pressure correction from the R858 algorithm is almost 2 decades in power below the uncorrected pressure, indicating that the correction has minimal adverse effect. Also noted is that the pressure shows whitening at frequencies higher than $1 \mathrm{~Hz}$. This noise is probably aerodynamic in origin with a standard deviation 


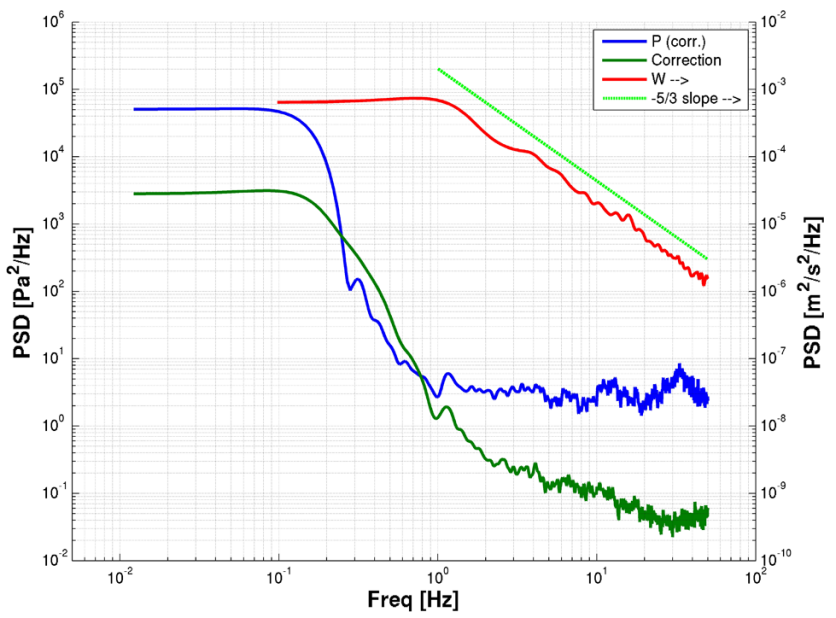

Fig. 13. Power spectral density [variance/frequency unit] versus frequency $[\mathrm{Hz}]$ for period of attack angle change at $7000 \mathrm{~m}$ altitude. Left axis: corrected static pressure [Pa], and pressure correction $[\mathrm{Pa}]$. Right axis: vertical wind component $\left[\mathrm{m} \mathrm{s}^{-1}\right]$ and inertial subrange $-5 / 3$ slope.

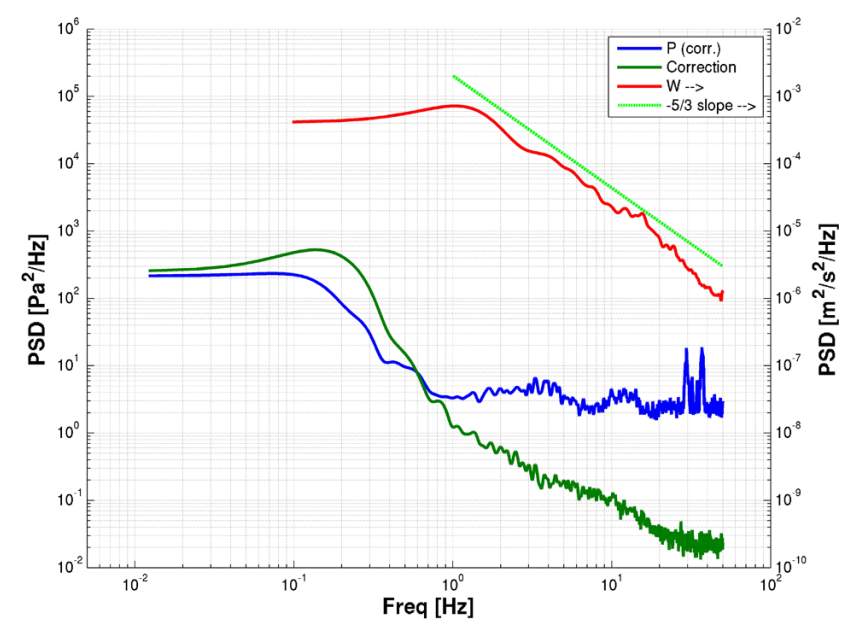

Fig. 14. Power spectral density as in Fig. 13 for period of sideslip angle changes at $7000 \mathrm{~m}$ altitude.

of about $15 \mathrm{~Pa}$, which is above the whitening effect of the digitization noise $(2 \mathrm{~Pa})$. The sharp disturbances at $30-40 \mathrm{~Hz}$ in Fig. 14 (sideslip periods) are probably due to the 4-bladed propellers, which operate normally at about $1700 \mathrm{rpm}$.

\section{Summary and discussion}

An algorithm was developed to estimate static pressure errors in steady flight using R858 differential pressure measurements, and tested with trailing cone data from a University of Wyoming King Air flight in 2005. After calibrating $f$ using the TC flight data, it was shown that the effects of speed, altitude and flight configuration (landing gear, flap extension) can be predicted to $\sigma=8 \mathrm{~Pa}$ in steady flight. To capture the effects of acceleration, flight maneuvers were conducted and geometric altitude from a GPS-aided inertial measurement data were used to predict the pressure error. These results suggest that pressure errors can be determined with a precision of \pm 20 Pa during such maneuvers. It should be emphasized that the precision, not the absolute accuracy, of these estimates is addressed in this paper. The absolute accuracy of the error estimates using this method depend on this empirical determination of $f$ as well as other factors addressed in the present work.

Our attempts to use statistical regression analysis alone to relate the observed pressure error to flight data ( $N_{\mathrm{Mach}}, q_{c}$, inertial acceleration) have not been successful. The differential pressure method, however, has the advantage of being a solution based upon the R858 equations, as presented in the Appendix. The main weakness is that determination of the probe sensitivity $f$ requires an independent means of its determination. In the present study, we used the TC measurements to calibrate $f$.

There are several sources of error which may be a factor in the interpretation. The effect of acceleration of the air in the connecting tubing, which we estimate to be smaller than $10 \mathrm{~Pa}$, is indistinguishable from the aerodynamic cause of the static defect at the sensing ports. Nonetheless, the R858 pressure imbalance approach should capture the connecting tubing effect. The other factor is the error introduced by uncertainty in height differences between the static pressure ports at the rear of the fuselage, IMU and GPS antenna locations, and R858 probe tip. Figure $8 \mathrm{~d}$ shows this effect is $<-15 \mathrm{~Pa}$.

Our flow modeling suggests that the relatively low estimates of $f$ from the algorithm $(f \cong 1.7$ ) may be reasonable since $f$ was found to decrease as the structure behind the R858 hemisphere-cylinder gets larger (Fig. 7). These values are lower than TR03 (hemisphere-cylinder, $f=2.07$ ) which also suggest that $f$ varies with incidence angle. It would be useful to obtain independent confirmation of these estimates, for example, by using pitch angle as a surrogate for $\alpha$ at differing airspeeds, as described by Brown et al. (1983). But that approach has limitations, as discussed in Sect. 2, especially since using pitch as an estimate of $\alpha$ implicitly incorporates the upwash effect (Crawford et al., 1996) into the sensitivity. Further, using a separate pitot tube measurement for $q$ would itself require the correction for static defect. The problems extend to the horizontal with sidewash effects. Thus, we think that independently estimating $f$, while desirable, is problematic at best and beyond the scope of this paper.

One possible shortcoming of the theoretical prediction of pressure distribution on the hemisphere, as shown in the Appendix, is the a priori assumption that $f$ is constant, while TR3 suggests that $f$ varies with attitude angle. Further, the flow modeling suggests that $f$ may be different for the vertical and lateral axes when the probe is ahead of an asymmetrical body like an aircraft fuselage and wing. The complete characterization of the probe would require a 3-dimensional flow modeling of the entire aircraft with the boom and probe. 
However, the precision with which our differential pressure method compares with the TC pressure reference and the IMU/GNSS altitudes belies a serious problem here.

We speculate that addition of a fifth differential pressure measurement to the R858 would eliminate $f$ as an unknown. We are currently studying the choices for a fifth pressure measurement that optimizes the ability to resolve static pressure errors, and planning that modification and evaluation for a future study.

\section{Appendix A}

\section{Derivation of differential pressure equations for a spherical 5-hole probe}

The derivation of the relationships among the pressures on a 5-hole spherical probe surface and the attack and sideslip flow angles follows. Hale and Norrie (1967), Brown et al. (1983), and Nacass (1992) analyzed the differential pressures between ports in terms of the well-known pressure distribution on a sphere in terms of the coefficient of pressure $C_{\mathrm{p}}$ :

$C_{\mathrm{p}}=\frac{P-P_{\infty}}{q}=1-f \sin ^{2} \phi$

where $P$ is the pressure at solid angle $\phi$ from the stagnation point, $P_{\infty}$ is the pressure in the free stream, $q \simeq 1 / 2 \rho U^{2}$ is the dynamic pressure, $\rho$ is the air density, $U$ the speed, and $f$ the sensitivity factor; $f=9 / 4$ for potential flow (Lamb, 1932).

A coordinate system is defined by unit vectors as follows: $\hat{i}$ along the $\mathrm{x}$-axis forward through the center port; $\hat{j}$ along the $\mathrm{y}$-axis to the right, and $\hat{k}$ long the z-axis down in aircraft coordinates. The angle $\phi$ in Eq. (A1) is the "great circle" angle between the stagnation point and point of pressure measurement at one of the five ports (Nacass, 1992). We define two more unit vectors in terms of their direction cosines from the probe axes: one, $\hat{\lambda}_{0}$, from the center of the probe hemisphere through the stagnation point, and the other, $\hat{\lambda}_{a}$, through the pressure port of interest. Thus,

$\hat{\boldsymbol{\lambda}}_{0}=\hat{\boldsymbol{i}} \cos \theta_{x 0}+\hat{\boldsymbol{j}} \cos \theta_{y 0}+\hat{\boldsymbol{k}} \cos \theta_{z 0}$

$\hat{\boldsymbol{\lambda}}_{a}=\hat{\boldsymbol{i}} \cos \theta_{x a}+\hat{\boldsymbol{j}} \cos \theta_{y a}+\hat{\boldsymbol{k}} \cos \theta_{z a}$

and the direction cosines for each vector are constrained by the identity

$\cos ^{2} \theta_{x}+\cos ^{2} \theta_{y}+\cos ^{2} \theta_{z}=1$.

Angle $\phi$ then can be found from the definition of the cross product of two vectors

$\sin \phi=\left|\hat{\lambda}_{a} \times \hat{\lambda}_{0}\right| /\left|\hat{\lambda}_{a}\right|\left|\hat{\lambda}_{0}\right|$ which can be expanded as

$$
\begin{aligned}
\sin ^{2} \phi & =\cos ^{2} \theta_{x a}\left(1-\cos ^{2} \theta_{x 0}\right)+\cos ^{2} \theta_{y a}\left(1-\cos ^{2} \theta_{y 0}\right) \\
& +\cos ^{2} \theta_{z a}\left(1-\cos ^{2} \theta_{z 0}\right) \\
& -2 \cos \theta_{x a} \cos \theta_{y a} \cos \theta_{x 0} \cos \theta_{y 0} \\
& -2 \cos \theta_{x a} \cos \theta_{z a} \cos \theta_{x 0} \cos \theta_{z 0} \\
& -2 \cos \theta_{y a} \cos \theta_{z a} \cos \theta_{y 0} \cos \theta_{z 0} .
\end{aligned}
$$

The coordinate system is defined with regard to aircraft axes as follows: $\mathrm{x}$-axis forward through the center port $1, \mathrm{y}$-axis right, and $\mathrm{z}$-axis down. Ports 2 (positive) and 3 are in $\mathrm{x}-\mathrm{y}$ plane, and ports 4 (positive) and 5 in the $\mathrm{x}-\mathrm{z}$ plane. The center port then has $\theta_{x a}=0^{\circ}, \cos \theta_{x a}=1$. The equations for $\phi$ for each port are then

$$
\begin{aligned}
\sin ^{2} \phi_{1} & =1-\cos ^{2} \theta_{x 0} \\
\sin ^{2} \phi_{2} & =\left(\cos \theta_{x 0} \cos \theta_{y 2}-\cos \theta_{y 0} \cos \theta_{x 2}\right)^{2}+\cos ^{2} \theta_{z 0} \\
\sin ^{2} \phi_{3} & =\left(\cos \theta_{x 0} \cos \theta_{y 3}-\cos \theta_{y 0} \cos \theta_{x 3}\right)^{2}+\cos ^{2} \theta_{z 0}(\text { A6 }) \\
\sin ^{2} \phi_{4} & =\left(\cos \theta_{x 0} \cos \theta_{z 4}-\cos \theta_{z 0} \cos \theta_{x 4}\right)^{2}+\cos ^{2} \theta_{y 0} \\
\sin ^{2} \phi_{5} & =\left(\cos \theta_{x 0} \cos \theta_{z 5}-\cos \theta_{z 0} \cos \theta_{x 5}\right)^{2}+\cos ^{2} \theta_{y 0}
\end{aligned}
$$

where the first subscript on the direction cosines is the axis direction, and the second subscript is the port number or 0 being the stagnation point.

Four differential pressures are measured: $P_{1}-P_{\infty}$ which approximately the impact pressure $q_{c}$ at small angles, $P_{2}-P_{3}$ in the plane of the probe horizontal axis defining the sideslip angle $\beta, P_{4}-P_{5}$ in the plane of the probe vertical axis defining the attack angle $\alpha$, and $P_{1}-P_{2}$ which is also a measure of the impact pressure, as suggested by Rosemount (1976) for their Model 858 5-hole probe. The center port then has $\theta_{x a}=0^{\circ}, \cos \theta_{x a}=1$, and the remaining ports are at $\theta=45^{\circ}, \cos \theta_{y a}=\cos \theta_{z a}=\sqrt{2} / 2$. Combining these angles and differential pressure definitions with Eq. (A6) applied to Eq. (A1) gives the following set of equations for the differential pressures:

$$
\begin{aligned}
& P_{1}-P_{\infty}=q\left[1-f\left(1-\cos ^{2} \theta_{x 0}\right)\right] \\
& P_{2}-P_{3}=2 q f \cos \theta_{x 0} \cos \theta_{y 0} \\
& P_{4}-P_{5}=2 q f \cos \theta_{x 0} \cos \theta_{z 0} \\
& P_{1}-P_{2}=\frac{1}{2} q f\left(\cos ^{2} \theta_{x 0}-\cos ^{2} \theta_{y 0}-2 \cos \theta_{x 0} \cos \theta_{y 0}\right)
\end{aligned}
$$

We now define the attack angle $\alpha$ and sideslip angle $\beta$ as functions of the velocity components in terms of the direction cosines $\left(U_{x} / U=\cos \theta_{x 0}\right.$, etc.):

$\tan \alpha=\frac{U_{z}}{U_{x}}=\frac{U \cos \theta_{z 0}}{U \cos \theta_{x 0}}=\frac{\cos \theta_{z 0}}{\cos \theta_{x 0}}$

$\tan \beta=\frac{U_{y}}{U_{x}}=\frac{U \cos \theta_{y 0}}{U \cos \theta_{x 0}}=\frac{\cos \theta_{y 0}}{\cos \theta_{x 0}}$. 
Note that $\beta$ as defined here is not the standard definition of sideslip (ISO, 1985), but is the commonly used definition because of its natural relation to $U_{y}$ in the wind computation.

Equations (A8) and (A9) can be solved for the direction cosines as

$$
\begin{aligned}
& \cos \theta_{x 0}=1 /\left(1+\tan ^{2} \alpha+\tan ^{2} \beta\right)^{1 / 2} \\
& \cos \theta_{y 0}=\tan \beta /\left(1+\tan ^{2} \alpha+\tan ^{2} \beta\right)^{1 / 2} \\
& \cos \theta_{z 0}=\tan \alpha /\left(1+\tan ^{2} \alpha+\tan ^{2} \beta\right)^{1 / 2} .
\end{aligned}
$$

Equations (A7)-(A10) can be combined to give the final set of equations, assuming exact knowledge of $P_{\infty}$ :

$$
\begin{aligned}
\Delta P_{1}=P_{1}-P_{\infty}= & q\left[1-(f-1)\left(\tan ^{2} \alpha+\tan ^{2} \beta\right) /\right. \\
& \left.\left(1+\tan ^{2} \alpha+\tan ^{2} \beta\right)\right] \\
\Delta P_{\alpha}=P_{4}-P_{5}= & 2 f q \tan \alpha /\left(1+\tan ^{2} \alpha+\tan ^{2} \beta\right) \\
\Delta P_{\beta}=P_{2}-P_{3}= & 2 f q \tan \beta /\left(1+\tan ^{2} \alpha+\tan ^{2} \beta\right) \\
\Delta P_{R}=P_{1}-P_{2}= & f q\left(1-2 \tan \beta-\tan ^{2} \beta\right) / \\
& 2\left(1+\tan ^{2} \alpha+\tan ^{2} \beta\right) .
\end{aligned}
$$

Equation (A11) can be solved either numerically or analytically for the unknowns $\tan \beta, \tan \alpha, q$ and $f$. The $\Delta P_{\alpha}, \Delta P_{\beta}$, and $\Delta P_{R}$ equations can be solved analytically $\tan \beta, \tan \alpha$ without a priori knowledge of dynamic pressure $q$ or sensitivity factor $f$. Those solutions are:

$$
\begin{aligned}
\tan \beta= & \left(\sqrt{2\left(\Delta P_{\beta}^{2}+2 \Delta P_{\beta} \Delta P_{R}+2 \Delta P_{R}^{2}\right)}\right. \\
& \left.-\Delta P_{\beta}-2 \Delta P_{R}\right) / \Delta P_{\beta} \\
\tan \alpha= & (\tan \beta) \Delta P_{\alpha} / \Delta P_{\beta} .
\end{aligned}
$$

We note that the limiting relationship when $\Delta P_{B} \rightarrow 0$ is

$\tan \alpha=\frac{\Delta P_{\alpha}}{4 \Delta P_{R}}$

and

$q=\frac{\Delta P_{\alpha}^{2}+8 \Delta P_{1} \Delta P_{R}}{8 \Delta P_{R}}$

Acknowledgements. This study was supported by NSF Cooperative Agreement AGS-0334908, and NSF Grant AGS-1034862. The authors would like to thank colleagues in the Department of Atmospheric Science at the University of Wyoming and the UWKA facility team for collection and processing of the data, and Prof. William Lindberg, UW Department of Mechanical Engineering, for assistance with the wind tunnel measurements and recording. We also would like to thank an anonymous reviewer for suggesting important improvements in the text, and also for noticing a typographical error in the equations in the Appendix.

Edited by: P. Herckes

\section{References}

Bellamy, J. C.: The use of pressure altitude and altimeter corrections in meteorology, J. Meteorol., 2, 1-79, doi:10.1175/15200469(1945)002<0001:TUOPAA>2.0.CO;2, 1945.

Bögel, W. and Baumann, R.: Test and calibration of the DLR Falcon wind measuring system by maneuvers, J. Atmos. Ocean. Tech., 8, 5-18, doi:10.1175/15200426(1991)008<0005:TACOTD>2.0.CO;2, 1991.

Brown, E. N.: Position Error Calibration of a Pressure Survey Aircraft Using a Trailing Cone, Tech. Rep. NCAR/TN313+STR, National Center for Atmospheric Research, Boulder, CO, doi:10.5065/D6X34VF1, 1988.

Brown, E. N., Shapiro, M. A., Kennedy, P. J., and Friehe, C. A.: The application of airborne radar altimetry to the measurement of height and slope of isobaric surfaces, J. Appl. Meteorol., 20, 171-180, doi:10.1175/15200450(1981)020<1070:TAOARA > 2.0.CO;2, 1981.

Brown, E. N., Friehe, C. A., and Lenschow, D. H.: The use of pressure fluctuations on the nose of an aircraft for measuring air motion, J. Clim. Appl. Meteorol., 22, 1070-1075, doi:10.1175/1520-0450(1983)022<0171:TUOPFO > 2.0.CO;2, 1983.

Crawford, T. L. and Dobosy, R. J.: A sensitive fast-response probe to measure turbulence and heat-flux from any airplane, Bound.Lay. Meteorol., 59, 257-278, doi:10.1007/BF00119816, 1992.

Crawford, T. L., Dobosy, R. J., and Dumas, E. J.: Aircraft wind measurement considering lift-induced upwash, Bound.-Lay. Meteorol., 80, 79-94, doi:10.1007/BF00119012, 1996.

Doebelin, E. O.: Measurement systems: application and design, McGraw-Hill, New York, 4th Edn., ISBN: 978-0070173385, 1990.

Hale, M. R. and Norrie, D. H.: The analysis and calibration the fivehole spherical pitot, Tech. Rep. ASME Publication 67-WA/FE24, Amer. Soc. Mech. Eng., New York, 1967.

Ikhtiari, P. A. and Marth, V. G.: Trailing cone static pressure measurement device, J. Aircraft, 1, 93-94, doi:10.2514/3.43563, 1964.

ISO: Flight dynamics, concepts and quantities Part 2: Motions of aircraft and the atmosphere relative to the earth, 2nd Edn., Ref. No. ISO 1151/2-1985(E), International Organization for Standardization, Geneva, Switzerland, 1985.

Lamb, H.: Hydrodynamics, Dover Publications, New York, ISBN: 978-0486602561, 1932.

LeMone, M. A. and Tarleton, L. F.: The use of inertial altitude in the determination of the convective-scale pressure field over land, J. Atmos. Ocean. Tech., 3, 650-661, doi:10.1175/15200426(1986)003<0650:TUOIAI>2.0.CO;2, 1986.

LeMone, M. A., Tarleton, L. F., and Barnes, G. M.: Perturbation pressure at the base of cumulus clouds in low shear, Mon. Weather Rev., 116, 2062-2068, doi:10.1175/15200493(1988)116<2062:PPATBO > 2.0.CO;2, 1988.

Mabry, G. and Brumby, R.: DC-8 Airspeed Static Position Error Repeatability, Tech. Rep. Douglas Paper 5517, Douglas Aircraft Co., Unk., 1968.

Nacass, P. L.: Theoretical Errors on Airborne Measurements of: Static Pressure, Impact Temperature, Air Flow Angle, Air Flow Speed, Tech. Rep. NCAR/TN-385+STR, National Center for Atmospheric Research, Boulder, CO, doi:10.5065/D6M61H79, 1992. 
Parish, T. R.: Forcing of the summertime low-level jet along the california coast, J. Appl. Meteorol., 39, 2421-2433, doi:10.1175/1520-0450(2000)039<2421:FOTSLL > 2.0.CO;2, 2000.

Parish, T. R. and Leon, D.: Measurement of cloud perturbation pressures using an instrumented aircraft, J. Atmos. Ocean. Tech., online first: doi:10.1175/JTECH-D-12-00011.1, 2012.

Parish, T. R., Rodi, A. R., and Clark, R. D.: A case study of the summertime Great Plains Low Level Jet, Mon. Weather Rev., 116, 94-105, doi:10.1175/15200493(1988)116<0094:ACSOTS > 2.0.CO;2, 1988.

Parish, T. R., Burkhart, M. D., and Rodi, A. R.: Determination of the horizontal pressure gradient force using Global Positioning System onboard an instrumented aircraft, J. Atmos. Ocean. Tech., 24, 521-528, doi:10.1175/JTECH1986.1, 2007.

Rodi, A. R. and Parish, T. R.: Aircraft measurement of mesoscale pressure gradients and ageostrophic winds, J. Atmos. Ocean. Tech., 5, 91-101, doi:10.1175/15200426(1988)005<0091:AMOMPG>2.0.CO;2, 1988.
Rosemount: Model 858 Flow Angle Sensors, Tech. Rep. Bulletin 1014, Revised 4/76, Rosemount Engineering, Minneapolis, MN, 1976.

Shapiro, M. A. and Kennedy, P. J.: Research aircraft measurements of jet stream geostrophic and ageostrophic winds, J. Atmos. Sci., 38, 2642-2652, doi:10.1175/15200469(1981)038<2642:RAMOJS > 2.0.CO;2, 1981.

Traub, L. and Rediniotis, O.: Analytic prediction of surface pressures over a hemisphere-cylinder at incidence, J. Aircraft, 40, 645-652, doi:10.2514/2.3168, 2003.

Wendisch, M. and Brenguier, J. (Eds.): Airborne Measurements for Environmental Research, Wiley-VCH Verlag $\mathrm{GmbH} \&$ Co. KGaA, Weinheim, Germany, ISBN: 978-3-527-40996-9, in press, 2013. 